\title{
Phase and coherence analysis of VHF scintillation over Christmas Island
}

\author{
E. B. Shume ${ }^{1}$, A. J. Mannucci $^{1}$, and R. Caton ${ }^{2}$ \\ ${ }^{1}$ Jet Propulsion Laboratory, California Institute of Technology, Pasadena CA, USA \\ ${ }^{2}$ Air Force Research Laboratory, Kirtland, NM, USA
}

Correspondence to: E. B. Shume (esayas.b.shume@jpl.nasa.gov)

Received: 28 October 2013 - Revised: 22 February 2014 - Accepted: 25 February 2014 - Published: 28 March 2014

\begin{abstract}
This short paper presents phase and coherence data from the cross-wavelet transform applied on longitudinally separated very high frequency (VHF) equatorial ionospheric scintillation observations over Christmas Island. The phase and coherence analyses were employed on a pair of scintillation observations, namely, the east-looking and westlooking VHF scintillation monitors at Christmas Island. Our analysis includes 3 years of peak season scintillation data from 2008, 2009 (low solar activity), and 2011 (moderate solar activity). In statistically significant and high spectral coherence regions of the cross-wavelet transform, scintillation observations from the east-looking monitor lead those from the west-looking monitor by about 20 to $60(40 \pm 20) \mathrm{min}$ (most frequent lead times). Using several years (seasons and solar cycle) of lead (or lag) and coherence information of the cross-wavelet transform, we envisage construction of a probability model for forecasting scintillation in the nighttime equatorial ionosphere.
\end{abstract}

Keywords. Ionosphere (ionospheric irregularities)

\section{Introduction}

Equatorial spread $F$ (ESF) comprises plasma density irregularity processes occurring at night in the equatorial and low-latitude ionosphere. A broadband spectrum of fieldaligned plasma density irregularities (ESF) is excited by ionospheric interchange instabilities (Hysell, 2000). The performance of signals from trans-ionospheric communication and navigation satellites is severely degraded by scintillation caused by the plasma irregularities (de La Beaujardiére and the C/NOFS Science Definition Team, 2004; de Paula et al., 2003). Forecasting equatorial spread $F$ (ESF) and scintillation, and studying the variabilities are, therefore, outstanding problems of equatorial aeronomy research.

In a recent publication, Shume and Mannucci (2013) have suggested that phase and coherence of the cross-wavelet transform of longitudinally separated L-band scintillation observations over equatorial South America can be developed into scintillation and ESF forecast instruments. They have applied the phase and coherence calculations on two pairs of scintillation observations: (1) São Luís and Rio Branco (whose longitudes are separated by $23^{\circ}$ ), and (2) Alta Floresta and Huancayo (whose longitudes are separated by $21^{\circ}$ ). They have reported that in statistically significant and high spectral coherence regions of the cross-wavelet transform, scintillation observations over São Luís (Alta Floresta) lead those of Rio Branco (Huancayo) by $\sim 2$ to $3 \mathrm{~h}$ with a $95 \%$ frequency. In other words, if L-band scintillation happens over São Luís (Alta Floresta), there is a $95 \%$ likelihood that scintillation will also occur to the west over Rio Branco (Huancayo) after $\sim 2$ to $3 \mathrm{~h}$ suggesting that a forecast can be made ahead of scintillation occurrences.

Ultimately our objective is to develop a data-driven global scintillation and ESF forecast database. With that in mind, in this paper, by expanding the phase and coherence technique applied over the South American sector (Shume and Mannucci, 2013), we have conducted further study in eastern Pacific longitude sectors, namely, over Christmas Island $\left(2.0^{\circ} \mathrm{S}, 202.6^{\circ} \mathrm{E}\right.$, dip latitude $1.78^{\circ} \mathrm{S}$; Fig. 1). In this sector, the geophysical conditions, the geomagnetic field geometry (magnetic declination, $\sim 10^{\circ} \mathrm{E}$ ), and characteristics of plasma irregularities are different when compared to the American sector (Tsunoda, 1985; Aarons, 1993; Su et al., 2010), and hence the impetus for investigating longitudinal 


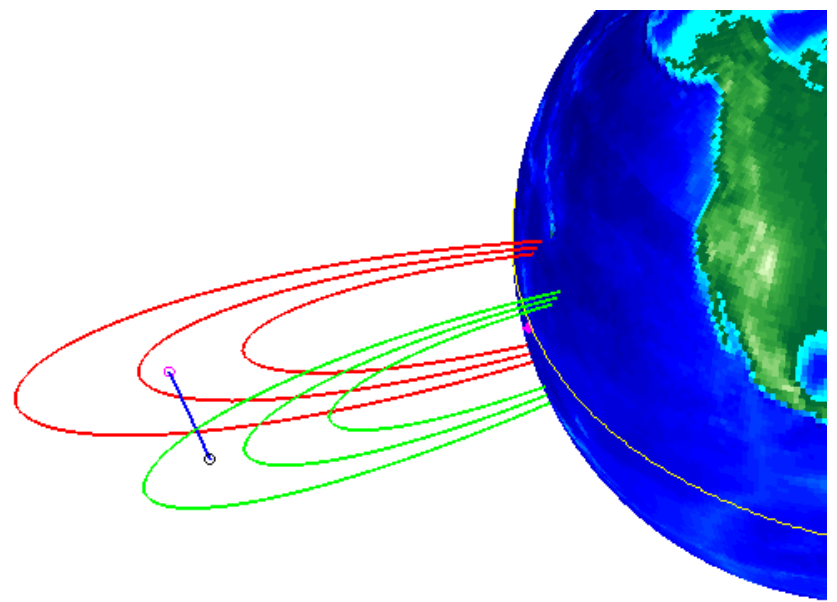

Fig. 1. The geometry of the VHF scintillation observations. Magenta circle shows the IPP $\left(1.70^{\circ} \mathrm{S}, 199.0^{\circ} \mathrm{E}\right)$ of the west-looking VHF signal monitor in a magnetic flux-tube context (field lines are shown as red and green, and were derived from the IGRF 11 model). The black circle shows IPP $\left(1.75^{\circ} \mathrm{S}, 208^{\circ} \mathrm{E}\right)$ of the east-looking monitor. The magnetic equator is shown by yellow line. The magenta star near the magnetic equator on the ground shows the location of the receiver.

properties of scintillation using the phase and coherence analysis technique.

In this short report, we specifically present coherence and phase of the cross-wavelet transform applied on the eastlooking and west-looking very high frequency (VHF) scintillation monitoring antennas over Christmas Island. The ionospheric pierce points (IPPs) of these two SATCOM (satellite communication, from geostationary satellite GEO) links are separated by about $9^{\circ}$ in longitude at $400 \mathrm{~km}$ altitude. (For further details about the coordinates of the IPPs of the east- and west-looking monitors, please consult Sects. 3.1 and 3.2.) The cross-wavelet analysis has been conducted in the context of building instruments for predicting scintillation and ESF plasma irregularities westward of scintillation observations. Application of coherence and phase calculations have been used in other geophysical applications such as climate research to forecast El Niño-Southern Oscillation (ENSO) and Indian summer monsoon (Torrence and Webster, 1999), and also for investigating the behavior of the Madden-Julian Oscillation (MJO) with respect to ENSO (Whitcher et al., 2000) (for example).

Equatorial plasma irregularities and scintillation forecasts are the main objective of the Communication/Navigation Outage Forecast System (C/NOFS) satellite mission (de La Beaujardiére and the C/NOFS Science Definition Team, 2004) and the Scintillation Network Decision Aid (SCINDA) network (Groves et al., 1997; Caton and Groves, 2006). We believe that the results of this research will contribute toward attaining the objectives of the C/NOFS satellite and SCINDA.

\section{Description of data and the analysis techniques}

\subsection{The scintillation index $S_{4}$ : definition and data source}

The scintillation index, $S_{4}$, is used as a quantitative measure of scintillation strength caused by plasma irregularities (Yeh and Liu, 1982; Kintner and Ledvina, 2005). The $S_{4}$ index is defined as the normalized standard deviation of signal intensity (Yeh and Liu, 1982):

$S_{4}^{2}=\frac{\overline{I^{2}}-\bar{I}^{2}}{\bar{I}^{2}}$,

where $I, \bar{I}$, and $\overline{I^{2}}$ represent the measured signal intensity, the average signal intensity, and the mean squared value of signal intensity, respectively.

To calculate coherence and phase of the east- and westlooking pair of scintillation monitors over Christmas Island (IPPs are separated by $\sim 9^{\circ}$ longitude), we have used 1 minute $S_{4}$ estimates of VHF signals transmitted from SATCOM (GEO satellites) and observed by the SCINDA network (Groves et al., 1997; Caton and Groves, 2006). The scintillation signals have been measured as the density irregularities convect zonally through fixed IPPs. The synchronized motion of the GEO satellites and the ground-based VHF receivers (SCINDA) facilitates the longitudinal localization of signal propagation path. In other words, the signal ray paths are fixed with respect to the receiver on the ground, which creates a suitable scenario for our analysis.

\subsection{Coherence and phase of the cross-wavelet transform}

Coherence of a cross-wavelet transform is defined as the covariance between one or more signals in a $2 \mathrm{D}$ timefrequency representation. Coherence is a parameter used to identify frequency bands and time intervals within which two signals exhibit common power. The two input signals for the cross-wavelet transform in this case are VHF scintillation $S_{4}$ observed by the east- and west-looking monitors over Christmas Island (Fig. 1, magenta color star).

Mathematically, the coherence function $R(a, b)$ can be described in terms of the cross-wavelet transform $W_{\phi_{i} \phi_{k}}(a, b)$ as follows (Sello and Bellazzini, 2000; Shume and Mannucci, 2013):

$R(a, b)=\frac{2\left|W_{\phi_{i} \phi_{k}}(a, b)\right|^{2}}{\left|W_{\phi_{i}}(a, b)\right|^{4}+\left|W_{\phi_{k}}(a, b)\right|^{4}}$,

where $0 \leq R(a, b) \leq 1$, and the cross-wavelet transform is defined as

$W_{\phi_{i} \phi_{k}}(a, b)=W_{\phi_{i}}(a, b) W_{\phi_{k}}^{*}(a, b)$.

$\phi_{i}$ and $\phi_{k}$ stand for distinct longitudes $i$ and $k$, and $*$ denotes complex conjugation. The parameters $a$ and $b$ are wavelet scale and translation factors, respectively. $W_{\phi_{i}}(a, b)$ stands 
for Fourier representation of a wavelet transform at longitude $\phi_{i}$ (Shume et al., 2013),

$$
W_{\phi_{i}}(a, b)=\int_{-\infty}^{+\infty} F\left[S_{4}(t)_{\phi_{i}}\right] \hat{\psi}^{*}(a \omega) e^{j \omega b} \mathrm{~d} \omega .
$$

Equation 4 represents the inverse Fourier transform of the product $F\left[S_{4}(t)_{\phi_{i}}\right] \hat{\psi}^{*}(a \omega)$, where $F\left[S_{4}(t)_{\phi_{i}}\right]$ is the Fourier transform of the scintillation signal $S_{4}(t)_{\phi_{i}}$ as a function of time $t$ around longitude $\phi_{i}$. In this analysis, we employed the Morlet wavelet, where $\hat{\psi}(a \omega)=(2 a \sqrt{\pi})^{\frac{1}{2}} e^{-\frac{1}{2}\left(a \omega-\omega_{\circ}\right)^{2}}$ and $\omega_{\circ}=6$.

The phase or the relative phase difference $P(a, b)$, in this specific case, produces a local measure of the delay of scintillation occurrences between the two longitudinally separated VHF signal monitors in a 2D time-frequency image highlighting a coherent behavior. The phase difference, which contains the lead/lag information, can be derived from the imaginary and real parts of the cross-wavelet transform $W_{\phi_{i} \phi_{k}}$ (Torrence and Webster, 1999; Shume and Mannucci, 2013):

$P(a, b)=\arctan \left(\frac{\Im\left(W_{\phi_{i} \phi_{k}}(a, b)\right)}{\Re\left(W_{\phi_{i} \phi_{k}}(a, b)\right)}\right)$,

where $-\pi \leq P(a, b) \leq \pi . P(a, b)>0$ indicates that longitude $\phi_{i}$ leads longitude $\phi_{k}$.

As a continuation of the research by Shume and Mannucci (2013), this study makes use of the coherence $R(a, b)$ and the phase $P(a, b)$ information of longitudinally separated VHF scintillation observation over Christmas Island. The phase and coherence information could be used to develop a global scintillation forecast system westward based on observations to the east. Statistically significant regions of the cross-wavelet spectra (compared to a background red-noise spectrum) will be mainly employed for our analysis and interpretations.

The analysis technique described above simultaneously determines the phase and coherence of scintillationproducing regions separated by longitude. As a result, the analysis provides phase (lead/lag) information of highly coherent structures with a measure of statistical significance. Computing the phase and coherence of longitudinally separated scintillation-producing regions could be important in light of the longitudinal connection of the regions by the large-scale wave structures (LSWSs).

\section{Analysis and interpretation}

\subsection{Ionospheric pierce points (IPPs)}

Figure 1 shows the geometry of the VHF scintillation observations over Christmas Island. The location of the VHF receiver is shown by the star (magenta color) that is close to the magnetic equator (yellow line). The figure shows the geographic coordinates of the IPPs (around $400 \mathrm{~km}$ altitude) of the east-looking (black circle) and west-looking (magenta circle) signal monitors relative to their corresponding magnetic flux tube. The magnetic equator is shown as a yellow curve, and has been derived from the IGRF-11 model. The blue line connecting the two IPPs is about $9.0^{\circ}$ in longitudinal extent (which is about $1000 \mathrm{~km}$ ).

The spatial locations of the IPPs in Fig. 1 do not change with time relative to their respective magnetic flux tubes while monitoring VHF signals (and scintillations) in concert transmitted from SATCOM. The plasma density perturbations in the sample volume of the IPPs, however, could change very rapidly with time as the density irregularities convect in the zonal direction.

\subsection{The data selection criteria for the phase and coherence analysis}

At least two longitudinally separated scintillation observations are required to do phase and coherence calculations. In this case we have used the east-looking and west-looking monitors whose IPPs are separated by about $9^{\circ}$ longitude as shown in Fig. 1.

The criteria we set to select the scintillation $S_{4}$ data for the phase and coherence analysis are the following:

1. Availability of at least 1 hour of continuous (without dropouts) VHF scintillation observations $S_{4}$,

2. Availability of simultaneous $S_{4}$ data observed by the east-looking and west-looking monitors,

3. The magnitude of the VHF scintillation index $S_{4}$ should be greater than 0.2 , and

4. Scintillation data free from radio frequency interference.

\subsection{Phase and coherence calculations}

The $S_{4}$ time series as a function of solar local time for 10 July 2008 over Christmas Island observed by the east-looking and west-looking signal monitors are shown in Fig. $2 \mathrm{a}$ and b, respectively. The corresponding power spectra (normalized by signal variance) are plotted in Fig. $2 \mathrm{c}$ and d exhibiting a common power around $160 \mathrm{~min}$ period. The coherence $R(a, b)$ (Eq. 2) and phase $P(a, b)$ (Eq. 5) of cross-wavelet transform of the two scintillation time series (shown in Fig. 2a and b) are shown in Fig. 2e and f.

The black contour curves, in Fig. 2e and f, enclose the statistically significant regions. The statistically significant regions are measured in terms of the ratio of the cross-wavelet spectral power to a background spectrum, in this case, a rednoise spectrum. The contour curves enclose regions where the cross-wavelet power spectrum is 2, 4, and 6 times larger than a red-noise spectrum, going from the outside contour 

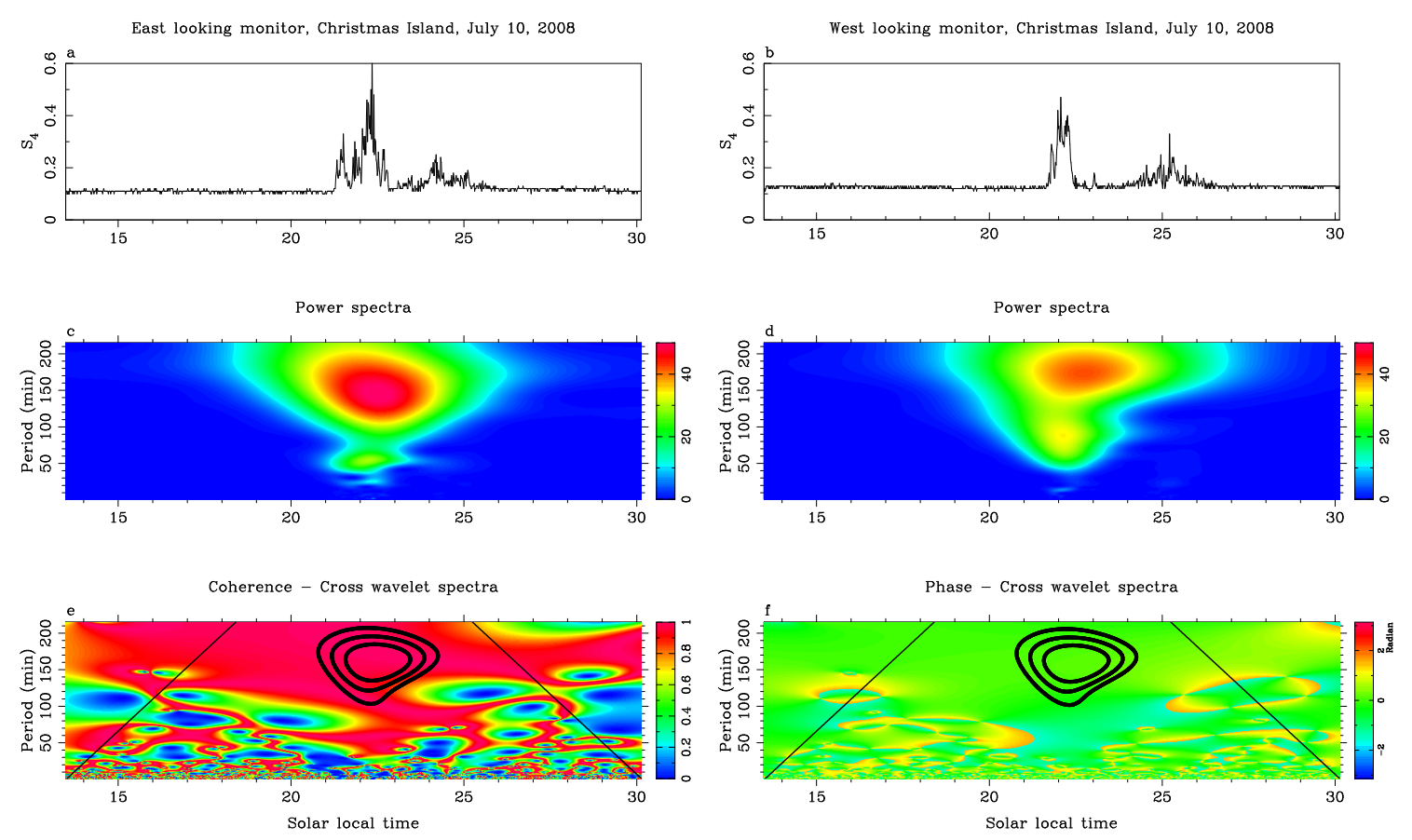

Fig. 2. (a) $S_{4}$ for the east-looking monitor on 10 July 2008, (b) $S_{4}$ for the west-looking monitor on 10 July 2008, (c) power spectra (normalized by signal variance) for the signal in (a), (d) power spectra (normalized by signal variance) for the signal in (b), (e) coherence of cross-wavelet transform for the east- and west-looking monitors, and (f) phase difference.

to the inside. In those regions the coherence is mostly $>0.8$ (Fig. 2e). In statistically significant regions (contour curves) and corresponding to high coherence, the phase difference (Fig. 2f) varies gently from about 0.1 to 0.3 radians showing a lead of scintillation occurrence for observations in the east compared to the west. The cones of influence (where edge effect becomes important) in Fig. 2e and $\mathrm{f}$ (shown by black solid lines) are far away from the region of interest.

We have repeatedly applied the phase and coherence calculation on 3 years of $S_{4}$ estimates from the east-looking and west-looking VHF signal monitoring pair, for the peak scintillation season periods in 2008, 2009, and 2011. In the west Pacific region, scintillation intensity peaks from June to October (Aarons, 1993). The 2010 scintillation data were severely contaminated with interference from unidentified sources. Based on the criteria set in Sect. 3.2, 35 days of $S_{4}$ from the peak season of 2008, 31 days of data from 2009, and 30 days of data from 2011 were selected for analysis. Horizontal cuts (about 10 min wide) containing the bands of frequencies through the statistically significant regions of the coherence and phase images (figures similar to Fig. 2e and $\mathrm{f}$ were generated) were made for the selected days. All the horizontal cuts of the coherences and phases (lead/lag) aggregated for the years 2008, 2009, and 2011 are plotted in Figs. 3, 4, and 5, respectively.

\subsection{Lead/lag information}

Figure 3a and b (2008), Fig. 4a and b (2009), and Fig. 5a and $b$ (2011) show the aggregate lead/lag and coherence as a function of time. Common features of the figures include the following: between about 19:00 and 23:00 (solar local time), the lead/lag time gravitates to 0.0 to $2.0 \mathrm{~h}$ and the coherence mostly exceeds 0.6 . The frequency of occurrence of lead/lag time and coherence are summarized in the histograms in Figs. 3c, d, 4c, d, 5c, and d. The histograms are produced using only a section of the coherence and lead/lag data between about 19:00 and 23:00. Overall there is a fair amount of similarity between the lead/lag (Figs. 3c, 4c, and 5c) and coherence (Figs. 3d, 4d, and 5d) distributions. The histograms (Figs. 3d, 4d, and 5d) show that the most frequent signal coherence values are $\geq 0.6$. Figures $3 c, 4 c$, and $5 \mathrm{c}$ show the frequency of occurrences of the lead/lag information in histograms for the years 2008, 2009, and 2011, respectively. The phase difference is converted to a lead/lag information based on the fact that the phase variations of $\pm \pi$ correspond to $\pm 12 \mathrm{~h}$. The common feature of the lead/lag histograms (Figs. 3c, $4 c$, and $5 c$ ) is that the most frequent lead times are concentrated between about 10 and around $60 \mathrm{~min}$ for IPPs separated by about $10^{\circ}$ longitudes (about $1000 \mathrm{~km}$ east-west distance).

Peak regions of the lead/lag histograms show that the scintillations observed by the east-looking sensor are ahead of scintillations observed by the west-looking sensor by about 

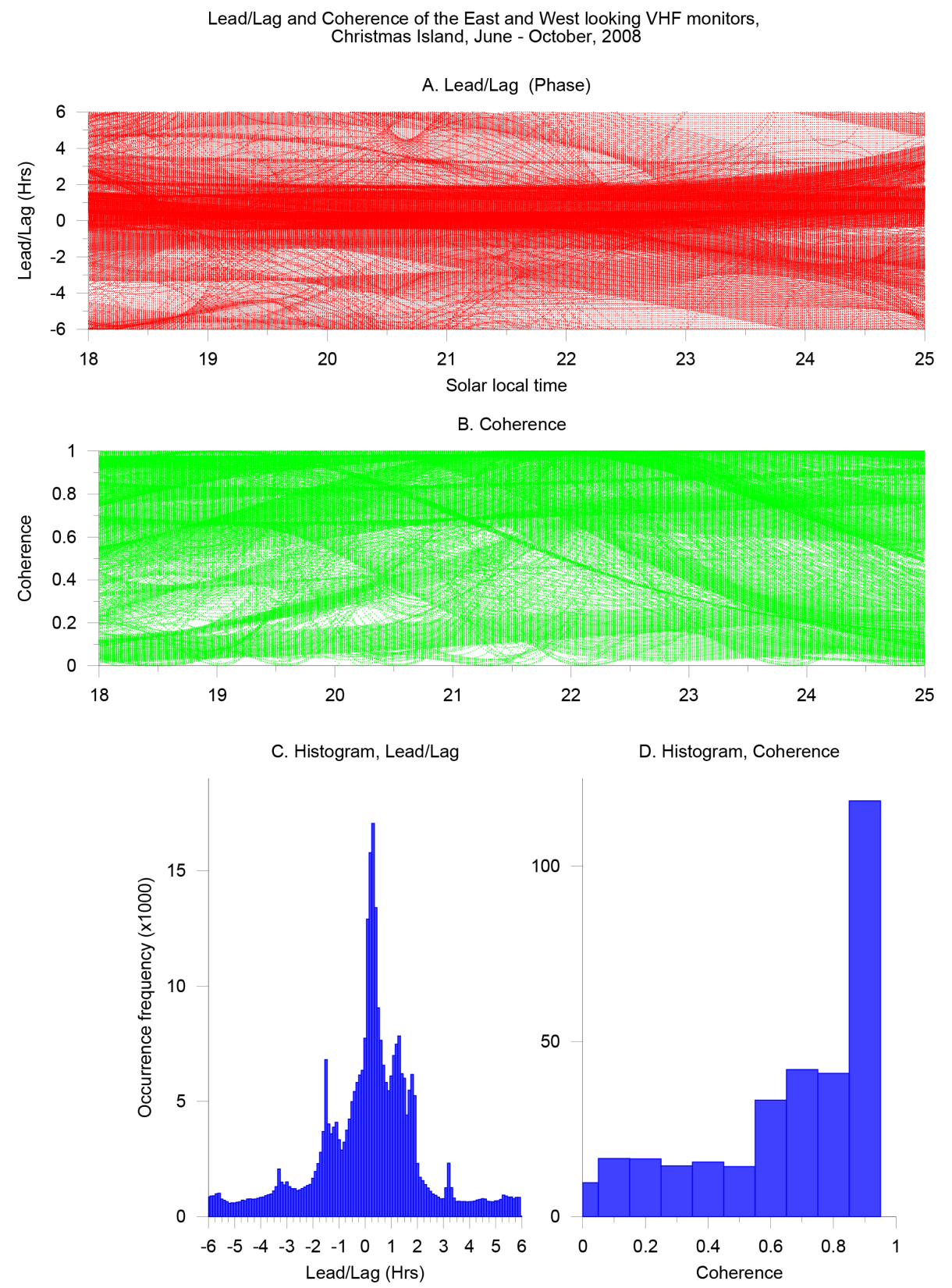

Fig. 3. (A) Lead/lag (phase difference) of the east- and west-looking VHF monitors, Christmas Island, for peak scintillation season 2008. (B) Coherence of the east- and west-looking VHF monitors, Christmas Island, 2008. (C) The histogram of the lead/lag (shown in Fig. 3a) distribution between the east-looking and west-looking VHF monitors in the statistically significant region over Christmas Island for 2008. (D) The histogram of coherence (shown in Fig. 3b) for peak scintillation season of 2008.

$30 \pm 10$ min (that means the lead ranges from 20 to $40 \mathrm{~min}$, Fig. 3c, 2008), by about $40 \pm 10 \mathrm{~min}$ (Fig. 4c, 2009), and by about $40 \pm 15 \mathrm{~min}$ (Fig. 5c, 2011). Lags (negative leads or phase) shown in the histograms correspond to mostly to a low coherence region. We also would like to point out that the lead/lag distribution has a relatively broader peak during the moderate solar activity year (2011) than the solar minimum years (2008 and 2009). This is perhaps due to extended presence of the irregularities during moderate solar activity conditions. The local sunset difference between the two IPPs (east- and west-looking antennas) is about $36 \mathrm{~min}$. This falls in the calculated 20 to $60 \mathrm{~min}$ lead times. However, in most cases, scintillation is generated well after local sunset (see Fig. $2 \mathrm{a}$ and $\mathrm{b}$, for example). Plasma instabilities and scintillation are generated after sunset when the local threshold geophysical conditions are convenient. The calculated 20 to 60 min lead times are determined by the complex geophysical system operating in the evening equatorial ionosphere. 

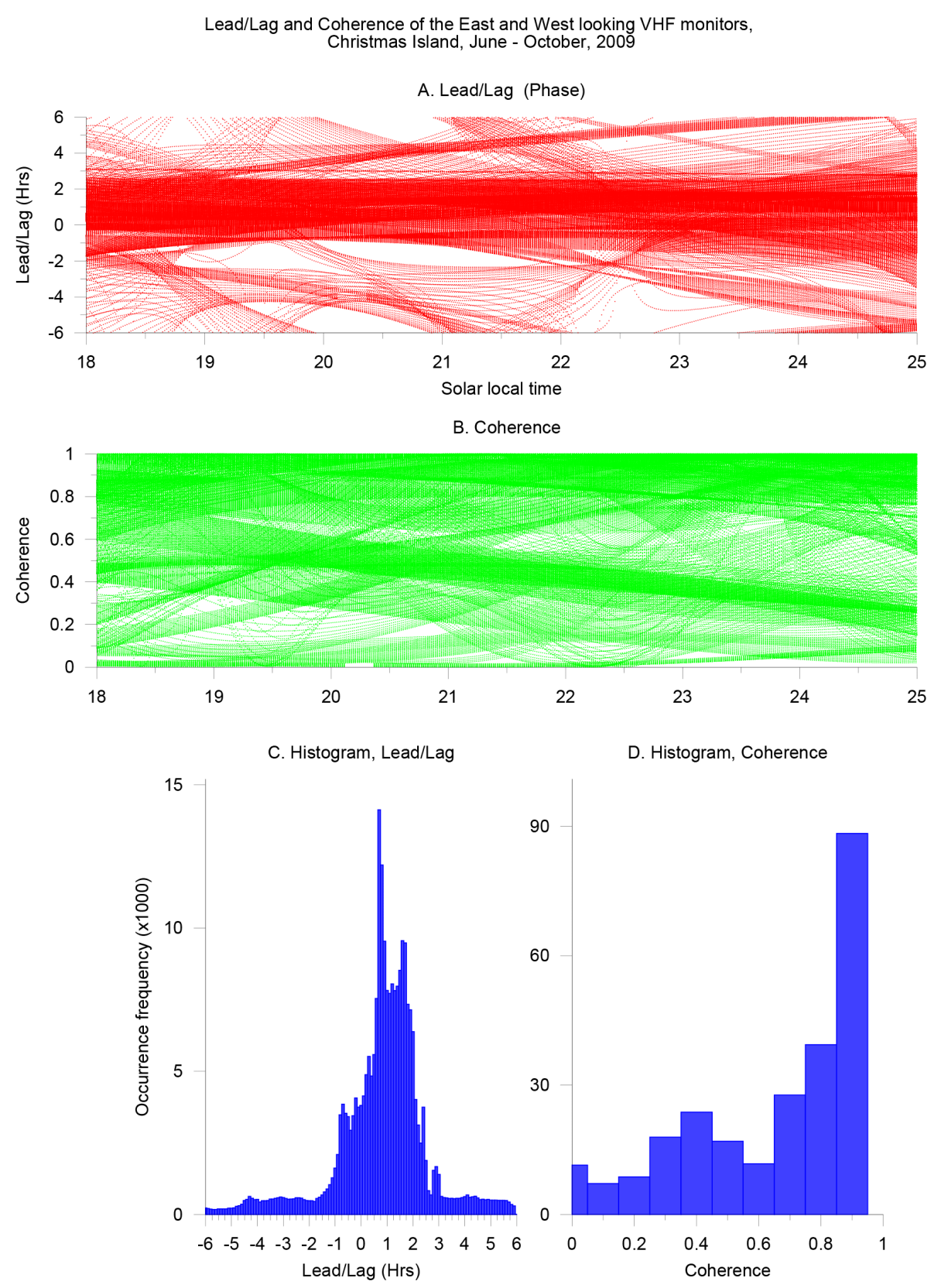

Fig. 4. Same as Fig. 3 but for peak scintillation season in 2009.

Practical and physical implications for scintillation prediction include the following: the main results of this paper indicate that, in statistically significant and high spectral coherence regions, VHF scintillation occurrences over the east of Christmas Island lead those of the scintillation occurrences over the west (about $1000 \mathrm{~km}$ to the west) by 20 to $60 \mathrm{~min}$. In other words, a prediction can be made about 20 to $60 \mathrm{~min}$ ahead of scintillation occurrences to the west. In addition, from several years of lead and coherence information, a global probability model for scintillation forecast can be constructed. The spread in the occurrence delays of about
$40 \pm 20$ (20 to 60) min implies different local time scintillation and plasma instability generation onsets to the west indicating that local sunset is not the only factor for the scintillation delay between the east and west. The various components of the geophysical system function in concert to generate scintillation that occurs with high likelihood in the west $40 \pm 20$ min after scintillation occurs in the east. 

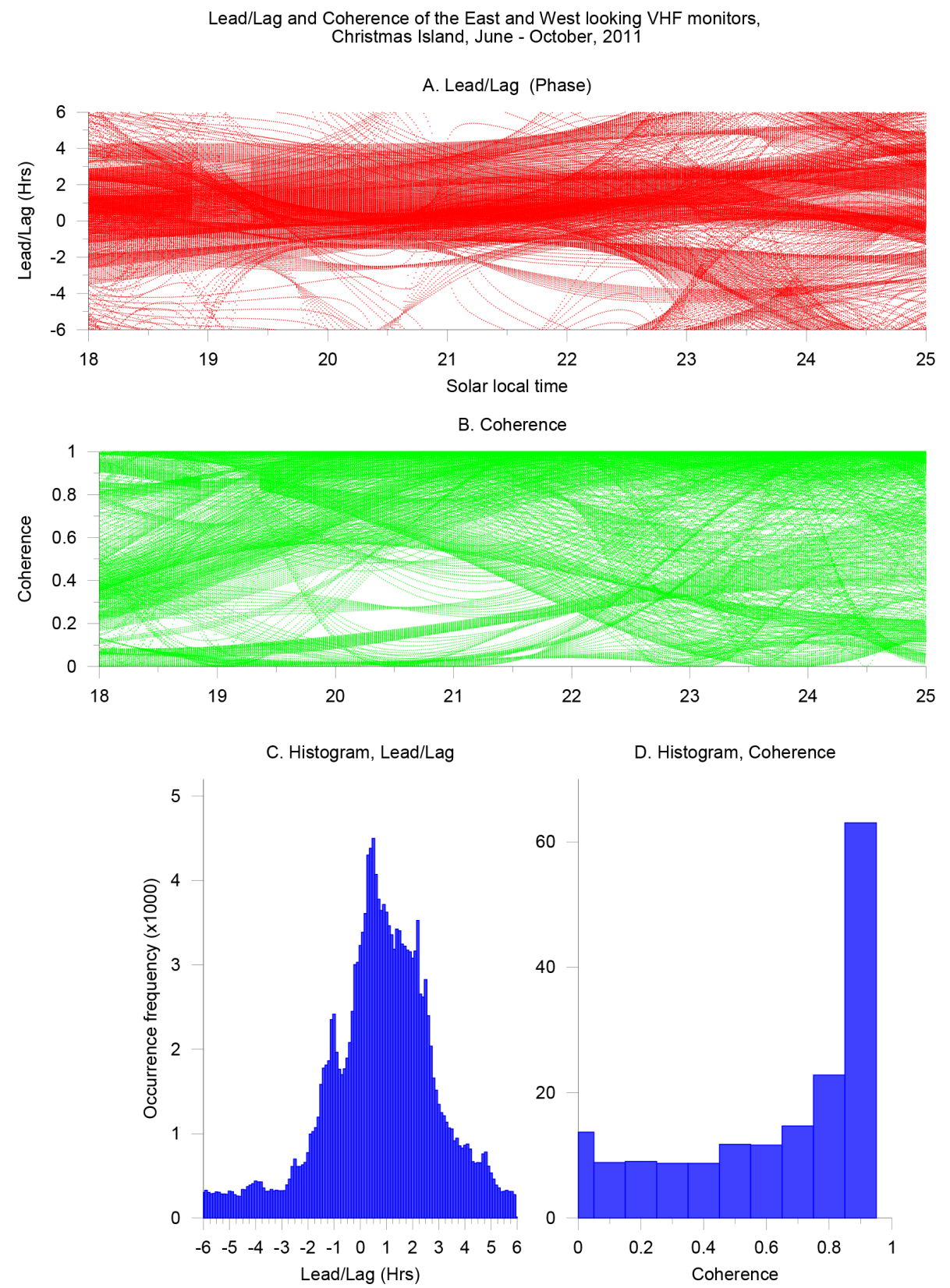

Fig. 5. Same as Fig. 3 but for peak scintillation season in 2011.

\section{Discussion}

A data driven investigation of longitudinal correlation of equatorial scintillation, which is related to the work at hand, has been reported by Caton and Groves (2006). Using longitudinally separated ground-based scintillation observations, they have investigated the longitudinal correlation of VHF and L-band scintillations over the nighttime equatorial ionosphere. They have concluded that significant correlation (> $40 \%$ ) of scintillation observations for longitudes separated by as much as $30^{\circ}$. In a recent publication, Shume and
Mannucci (2013) have reported that L-band scintillation occurrences over São Luís (Alta Floresta) lead those of Rio Branco (Huancayo) by about 2 to $3 \mathrm{~h}$ during equinox seasons (September to November 2012, and March to April 2012) these equatorial locations in South America are separated by about $20^{\circ}$ longitudes. The results presented in this paper, for VHF scintillation observed over Christmas Island, confirm also that global scintillation forecasting strategy can be built using longitudinally separated scintillation observations. Our results provide a measure of statistical significance of the calculated coherent and phase values. 
The phase (lead/lag) and coherence analysis technique shows that the longitudinally separated scintillationproducing regions are phase locked and are mutually coherent ionospheric plasma density irregularity structures. The longitudinally separated scintillation-producing regions might be connected by large-scale wave structures (for example, Thampi et al., 2009; Tsunoda et al., 2011), which are reported to be related to the generation of equatorial spread $F$ and scintillation. At the moment we are carrying out research to investigate the relationship between the LSWSs and lower atmospheric waves propagating from below using CERTO beacon scintillation data from the C/NOFS satellite (de La Beaujardiére and the C/NOFS Science Definition Team, 2004) thereby to understand the underlying mechanism of generating equatorial spread $F$ irregularities and scintillation.

\section{Summary and conclusions}

This report presents phase and coherence of VHF scintillation observations carried out over Christmas Island. VHF scintillation data from the peak seasons (June to October) of 2008, 2009, and 2011 observed by the east-looking and west-looking monitors have been selected to do the analysis. Our analysis has shown that, in statistically significant regions with high cross-spectral coherence, VHF scintillation occurrences over the east of Christmas Island lead those of the scintillation occurrences over the west (about $1000 \mathrm{~km}$ to the west) by 20 to $60 \mathrm{~min}$ suggesting a forecast can be made ahead of scintillation occurrences. The phase and coherence of cross-wavelet transform is instrumental to build global scintillation forecast system westward of observation sites based on scintillation observations to the east.

Acknowledgements. The research was carried out at JPL, California Institute of Technology, under a contract with NASA. E. Shume thanks the NPP administered by ORAU under a contract with NASA.

Topical Editor J. Klenzing thanks E. de Paula and one anonymous referee for their help in evaluating this paper.

\section{References}

Aarons, J.: The longitudinal morphology of equatorial $F$ layer irregularities relevant to their occurrence, Space Sci. Rev., 63, 209243, 1993.

Caton, R. and Groves, K.: Longitudinal correlation of equatorial ionospheric scintillation, Radio Sci., 41, RS5S22, doi:10.1029/2005RS003357, 2006.

de La Beaujardiére, O. and the C/NOFS Science Definition Team: C/NOFS: a mission to forecast scintillations, J. Atmos. Sol.-Terr. Phys., 66, 1573-1591, 2004. de Paula, E. R., Rodrigues, F. S., Iyer, K. N., Kantor, I. J., Abdu, M. A., Kintner, P. M., Ledvina, B. M., and Kil, H.: Equatorial anomaly effects on GPS scintillations in Brazil, Adv. Space Res., 31, 749-754, 2003.

Groves, K. M., Basu, S., Weber, E. J., Smitham, M., Kuenzler, H., Valladares, C. E., Sheehan, R., MacKenzie, E., Secan, J. A., Ning, P., McNeill, W. J., Moonan, D. W., and Kendra, M. J.: Equatorial scintillation and systems support, Radio Sci., 32, 2047-2064, 1997.

Hysell, D. L.: An overview and synthesis of plasma irregularities in the equatorial spread F, J. Atmos. Sol.-Terr. Phys., 62, 10371056, 2000.

Kintner, P. M. and Ledvina, B. M.: The ionosphere, radio navigation, and global navigation satellite systems, Adv. Space Res., 35, 788-811, 2005.

Sello, S. and Bellazzini, J.: Wavelet Cross-Correlation Analysis of Turbulent Mixing from Large-Eddy-Simulations, 41 - Mathematical and Physical Models, Enel Research, arXiv:physics/0003029 [physics.flu-dyn], 2000.

Shume, E. B. and Mannucci, A. J.: First calculation of phase and coherence of longitudinally separated L-band equatorial ionospheric scintillation, Geophys. Res. Lett., 40, 3496-3501, doi:10.1002/grl.50702, 2013.

Shume, E. B., Mannucci, A. J., Butala, M. D., Pi, X., and Valladadres, C. E.: Flux-tube analysis of L-band ionospheric scintillation, J. Geophys. Res., 118, 3791-3804, doi:10.1002/jgra.50285, 2013.

Su, S.-Y., Chen, M. Q., Chao, C. K., and Liu, C. H.: Global, seasonal, and local time variations of ion density structure at the low-latitude ionosphere and their relationship to the postsunset equatorial irregularity occurrences, J. Geophys. Res., 115, A02309, doi:10.1029/2009JA014339, 2010.

Thampi, S. V., Yamamoto, M., Tsunoda, R. T., Otsuka, Y., Tsugawa, T., Uemoto, J., and Ishii, M.: First observations of large-scale wave structure and equatorial spread $\mathrm{F}$ using CERTO radio beacon on the C/NOFS satellite, Geophys. Res. Lett., 36, L18111, doi:10.1029/2009GL039887, 2009.

Tsunoda, R. T.: Control of the seasonal and longitudinal occurrence of equatorial scintillations by the longitudinal gradient in integrated E region Pedersen conductivity, J. Geophys. Res., 90, 447-456, doi:10.1029/JA090iA01p00447, 1985.

Tsunoda, R. T., Yamamoto, M., Tsugawa, T., Hoang, T. L., Tulasi Ram, S., Thampi, S. V., Chau, H. D., and Nagatsuma, T.: On seeding, large-scale wave structure, equatorial spread $\mathrm{F}$, and scintillations over Vietnam, Geophys. Res. Lett., 38, L20102, doi:10.1029/2011GL049173, 2011.

Torrence, C. and Webster, P. J.: Interdecadal Changes in the ENSOMonsoon System, J. Climate, 12, 2679-2690, 1999.

Whitcher, B., Guttorp, P., and Percival, D. B.: Wavelet analysis of covariance with application to atmospheric time series, J. Geophys. Res., 105, 14941-14962, doi:10.1029/2000JD900110, 2000.

Yeh, K. C. and Liu, C.: Radio wave scintillations in the ionosphere, P. IEEE, 70, 324-360, 1982. 